RUNNING HEAD: ANARCHIC HAND WITH ABNORMAL AGENCY

\title{
Anarchic hand with abnormal agency following right inferior parietal lobe
}

damage: A case report

Paul M. Jenkinson ${ }^{1 *}$, Nicola M. J. Edelstyn ${ }^{2}$, Catherine Preston ${ }^{3}$ \& Simon J. Ellis ${ }^{4}$

${ }^{1}$ Department of Psychology, University of Hertfordshire, UK.

${ }^{2}$ School of Psychology, Keele University, UK.

${ }^{3}$ Department of Neuroscience, Karolinska Institute, Sweden.

${ }^{4}$ Department of Neurology, University Hospital of North Staffordshire, UK.

*Correspondence concerning this article should be addressed to: P. M. Jenkinson,

Department of Psychology, University of Hertfordshire, College Lane, Hatfield, AL10 9AB. Tel: 01707 284618. Email: p.jenkinson@herts.ac.uk. 


\section{Acknowledgements}

The authors are grateful to LX for her time, effort and patience; to the technical staff at Keele University who provided support in the experiment; and to Farrah Allarakha for her assistance with data collection. The authors disclose no conflicts of interest in the production of this work. 


\begin{abstract}
Anarchic hand syndrome (AHS) is characterised by goal-directed movements performed without volitional control (agency). Different AHS subtypes have been identified; however, few studies have examined the posterior subtype. We report a case of AHS following right-hemisphere parietal damage, with left-sided somatosensory and proprioceptive impairment. Agency was examined for nonanarchic (volitional) movements performed using the anarchic hand. The patient experienced abnormal agency for movements whether motor intention and visual feedback were congruent or incongruent, but not when intention was absent (passive movement). Findings suggest a general disturbance of veridical motor awareness and agency in this case of parietal AHS.
\end{abstract}

Abstract $=98$ words $(\max 100)$

Keywords: anarchic hand; agency; motor awareness; parietal lobe. 


\section{Introduction}

Anarchic hand syndrome (AHS) is a neurological condition in which complex, goal-directed (i.e. purposeful) movements of an upper limb occur without conscious volition. The condition has been subject to considerable confusion in scientific and popular literature, with the term 'alien limb' being applied to a range of different signs and symptoms, including the phenomenon currently recognised as AHS (described above; see Marchetti \& Della Sala, 1998, for a detailed discussion). An unfortunate consequence of this confusion has been an intrinsic difficulty revealing the neuroanatomical and functional bases of AHS. However, contemporary research distinguishes between AHS (as described above) and alien limb (a feeling that the hand is foreign and failure to recognise the ownership of one's limb; Marchetti \& Della Sala, 1998) based on now well-established subcomponents of selfconsciousness, namely: agency (i.e. the sense that I am the author or controller of my actions), and body ownership (i.e. the sense that my body belongs to me; see also Synofzik, Vosgerau \& Newen, 2008 for further discussion). Thus, alien hand currently describes a disturbance of body ownership, while AHS refers to a disturbance of the agency system deficit in the sense of agency.

Historically, AHS has been reported as most frequently occurring following damage to the corpus callosum and the supplementary motor area (SMA), with involuntary actions thought to arise from a failure to inhibit actions elicited by external cues (see Kritikos, Breen \& Mattingley, 2005; Riddoch, Humphreys \& Edwards, 2001). However, a parietal variant of AHS has also been identified, involving damage or disconnection of the parietal lobes (Graff-Radford et al., 2013; Scepkowski \& Cronin-Golomb, 2003). Although initially thought of as atypical, recent evidence suggests that parietal AHS, particularly involving the right 
hemisphere, is relatively common. For instance, Scepkowski and Cronin-Golomb (2003) review more than 50 published cases of 'alien hand' (referring to autonomous, complex movements of the upper limb performed against the patient's will), with specific consideration of the recently described subtype arising from damage to the parietal lobe or other posterior brain areas. Their review contains descriptions of 17 such cases; however, only two cases involved unilateral damage restricted to the parietal lobe following stroke, neither of which involved the right hemisphere, as described in the current case report (see Scepkowski \& Cronin-Golomb, 2003, p.274, Table 2).

More recently, Graff-Radford et al. (2013) examined the alien limb phenomenon (i.e. when a patient experiences that an extremity "is foreign" or "has a will of its own", together with observable involuntary motor activity, p.1881), in a retrospective analysis of medical records from patients seen in a neurology department between $1^{\text {st }}$ January 1996 and $11^{\text {th }}$ July 2011. They identified 150 patients with alien [i.e. anarchic] limb, 14 of which were a result of cerebrovascular events (10 right hemisphere, 4 left hemisphere). One of their most significant findings was the involvement of the parietal lobe in all 14 of these cases. Moreover, three cases involved damage restricted to the parietal lobe (cases 3, 9 \& 10, Graff-Radford et al., 2013, p. 1886, Table 4), indicting a key role of the right parietal lobe in the phenomenon.

Despite this recent recognition of parietal-type AHS as a relatively frequent occurrence, there remain relatively few empirical studies. One notable exception is an functional magnetic resonance imaging (fMRI) study of a patient with extensive right parietal lobe damage, who performed spontaneous (flexion-extension) movements of the left hand (fingers) without conscious will (Assal, Schwartz \& Vuilleumier, 2007). 
Movements performed without conscious volition were found to correspond with isolated activity in the primary motor cortex (M1), whereas voluntary movements of the same limb involved widespread bilateral activity in the parietal and premotor areas. Thus, parietal AHS might be explained by an uncoupling or bypassing of distributed volitional networks from regions responsible for motor production (i.e. M1).

This theoretical explanation of AHS is consistent with the established role of the parietal lobe in a multitude of sensorimotor functions relating to the body, movement and self-awareness (Sirigu et al., 2004). In particular, Desmurget and Sirigu (2009) propose a functional neuroanatomical model identify the posterior parietal cortex as important in motor awareness (i.e. being conscious of our own movements), claiming that conscious motor intentions and predictions regarding the sensory consequences of an action are generated or monitored in posterior parietal areas. These predictive processes (as opposed to afferent sensory information) give rise to our everyday, subjective awareness of movement. A parallel process of lowlevel (unconscious) comparison between actual and expected sensory information is performed in premotor areas during motor execution. These premotor areas allow errors of limited magnitude to be automatically and unconsciously corrected via internal feedback loops; however, an error signal is generated to draw conscious attention to errors (i.e. veridical motor awareness) when they are too large to be corrected without conscious awareness.

This account of the parietal lobe is supported by neuropsychological studies, as well as functional neuroimaging and behavioural experiments in healthy people. Other pathologies of motor awareness, such as anosognosia for hemiplegia (i.e. unawareness of paralysis) visuospatial neglect, and delusions of control (passivity) are 
well established as being associated with abnormalities in fronto-parietal networks (Berti et al., 2005; Frith, Blakemore \& Wolpert, 2000; Preston, Jenkinson \& Newport, 2010). Similarly, functional neuroimaging of healthy participants during simulated anarchic movements (i.e. experimentally induced mismatches between motor intention and visual feedback) reveal activation of a right fronto-parietal network (Leube, Knoblich, Erb \& Kircher, 2003). The frontal component is believed to detect mismatches between own actions and visual consequences, while the parietal component is thought to be involved in a more complex attribution process, such as when the distinction between oneself and another is critical and ambiguous during goal-directed movements. This is further supported by neuroimaging studies of motor awareness, which employ self-other discrimination tasks in healthy participants to examine the sense of agency. These studies reveal increased activity of the right inferior parietal lobe during 'other’ judgments (Farrer et al., 2003; Farrer \& Frith, 2002). By contrast, motor awareness and the sense of agency are impaired for selfother attributions of goal-directed movements when transcranial magnetic stimulation (TMS) is used to disrupt the right inferior parietal lobe (Preston \& Newport, 2008). This disruption of the inferior parietal lobe leads to an (apparently counterintuitive) increase in 'other' judgments (cf. Farrer and colleagues, 2002, 2003), which can be explained in terms of an interruption to the generation and monitoring of motor intentions and predictions, which form the basis of normal motor awareness (see Preston \& Newport, for further discussion).

Despite this wealth of evidence linking the parietal lobe with awareness of volitional movement, and the increasing recognition of a parietal subtype of AHS, experimental studies of parietal AHS remain scarce. Such studies are necessary to develop a clear nosology and understanding of the functional and neural mechanisms 
underpinning different types of AHS (Scepkowski \& Cronin-Golomb, 2013). We therefore report here a novel case of AHS following damage to the right inferior parietal lobe. Given the proposed role of the parietal lobe in motor awareness (Desmurget \& Sirigu, 2009), and evidence regarding the specific role of the inferior parietal lobe in attribution of goal-directed actions and agency (Farrer et al., 2003; Farrer \& Frith, 2002; Preston \& Newport, 2008), we expected these functions to be impaired in our patient (LX). Specifically, in line with the theory of Desmurget and Sirigu (2009), and findings of Preston and Newport (2008), we hypothesised that damage to the inferior parietal lobe would impair the usual mechanisms by which conscious motor intentions and sensory predictions are generated and monitored. We predicted that this impairment would lead to reduced self-reports of agency during active (i.e. volitionally generated by the participant / efferent motor signal present), goal-directed movements of the affected limb, regardless of whether motor intention and visual feedback were congruent or incongruent. Neurologically intact participants, on the other hand, would only deny agency when feedback was incongruent and thus did not accurately represent their actions. We further predicted that passive movements (i.e. generated by the experimenter / efferent motor signal absent) would result in negative agency judgments, in line with controls, and the established finding that efferent motor signals are necessary for the sense of agency to emerge in such tasks (see Tsakiris, Longo \& Haggard, 2010). The current study therefore assessed the subjective sense of agency for (active and passive) movements performed by the left (anarchic) arm of patient LX and healthy controls, under conditions of sensorimotor congruence and incongruence.

\section{Method}




\section{Case report}

At the time of testing LX was a 26-year-old right-handed woman. She was diagnosed with relapsing-remitting multiple sclerosis at age 23, and placed on monthly intravenous infusions of Tysabri ${ }^{\circledR}$ (natalizumab). Two weeks following the seventh infusion LX developed a venous sinus thrombosis with venous infarction and haemorrhage into her right parietal lobe (Figure 1a). A second MRI performed shortly after the study showed her brain to be free from sclerotic lesions (Figure 1b). Her symptoms immediately following the stroke included a complete loss of sensation, body positioning and temperature on the left side, extrapersonal neglect, and the occurrence of non-volitional (anarchic) movements of the left arm.

A clinical examination of LX performed by SJE revealed normal tone and power in all four limbs. A moderate left inferior homonymous quadrantinopsia was revealed on visual field testing. Although pinprick perception is lost on the right side of her face she continues to feel light touch, whereas both are lost on the left side of her face. Temperature perception is perceived on the right cheek only and lost from all four limbs. Vibration sensation is lost in the left hand but present in the other limbs. Joint perception is present for large excursions of the right hand and foot but lost for small excursions of the fingers and toes. In the left arm there is no perception of joint position at the fingers, wrist or elbow, and at the shoulder she only perceives movement because of the way it affects the rest of her body. LX also reported experiencing complex, goal-directed movements of the left arm which occurred against her will, stating that "at times my left hand grabs hold of things and keeps 
holding them... without meaning to do this” and that her left arm would scare her, because of the surprise movements it made. Examples of these behaviours included the arm sometimes pulling her hair or "strangling” her during the night, and taking food out of the shopping trolley in the supermarket. Despite the arm feeling "strange", LX never explicitly denied ownership of the arm, although she sometimes referred to it in the third person ("it will close doors...or pick pens up on its own”).

A brief neuropsychological assessment identified LX’s current IQ as 106 (Wechsler Abbreviated Scale of Intelligence; Wechsler, 1999), suggesting some decline compared to premorbid levels, which were estimated as 112 (National Adult Reading Test; Nelson and Willison, 1991). LX showed no sign of personal neglect (comb and razor/compact test; McIntosh, Brodie, Beschin \& Robertson, 2000), but some extrapersonal neglect (star cancellation test; Halligan, Marshall \& Wade, 1989). Materials and Procedure

Insert Figure 2 around here

The experiment was performed by LX and eight right-handed healthy controls (HCs) (2 male, mean age $=22.50, \mathrm{SD}=1.41$ ). The study was approved by a local ethics committee, and conducted in accordance with the declaration of Helsinki.

Participants sat in front of a metal frame $\left(1 \mathrm{~m}^{2}\right)$ that housed a video camera (see Figure $2 \mathrm{a})$. To the left of the frame ( $\sim 25 \mathrm{~cm}$ from the real hand) was a vertically positioned 28” LED monitor. The video camera (shutter speed = 25 frames/s; overall temporal delay $=42 \mathrm{~ms}$ ) was fixed to the frame behind a black curtain and connected to the display, such that when participants placed their hand through the curtain a lifesized image of the hand was viewed on the display, but not seen directly. The image 
provided a person-centred view of the hand as seen from above. A close match between the hand on the computer screen and real hand size was achieved by measuring the distance from the tip of the forefinger to the base of the palm on the real hand, and then using the camera zoom to adjust the screen image until these measurements corresponded. The visual display was located to the left of the real hand due to physical restrictions in the workspace; however, LX's ability to see the image, despite mild left-sided neglect, was checked before each condition.

Visual feedback and motor intention were manipulated across four conditions (Figure 2b) completed in a pseudorandom order. In the False-Moving condition participants were instructed not to move their hand but received false visual feedback of their index finger moving (lifting/extension and lowering/flexion in a steady manner for 90s). The magnitude of the finger lift (extension-flexion) movement was $\sim 6 \mathrm{~cm}$. False feedback was achieved by playing a pre-recorded video of the hand, which was obtaining surreptitiously during an initial 'practice' phase. In the FalseStill condition participants were instructed to lift and lower their index finger for 90s whilst provided with false visual feedback of their hand remaining motionless (prerecorded video of their stationary hand). In the Veridical condition participants were instructed to move their index finger and given veridical visual feedback. Finally, in the Passive condition participants were instructed to rest their hand whilst receiving veridical visual feedback of their finger being moved passively by the experimenter (lifting and lowering the finger via a piece of string attached to a ring worn on the participant's index finger). After each condition participants completed a six-item self-report questionnaire adapted from Tsakiris et al. (2010), assessing agency, body ownership/recognition, susceptibility (Control 1) and felt limb position (Control 2) (see Figure 3). Participants responded orally using a 7-point Likert scale $(-3=$ 
‘strongly disagree', +3 = 'strongly agree’).

\section{Results}

Insert Figure 3 around here

Overall measures of agency and ownership were produced by calculating the mean of the two statements previously identified as relevant for each measure. The two control questions (measuring susceptibility and limb position) were examined individually (since they examine different constructs).

Due to the HC data being ordinal and non-normally distributed, LX's performance was compared with HCs via bootstrapped 95\% confidence intervals (CI) (DiCiccio \& Efron, 1996). Figure 3c shows that LX's agency ratings fell outside the HC CIs in all conditions except Passive. LX never expressed a strong sense of agency during the experiment, with scores ranging between +1 and -0.5 . Her ratings during the Veridical condition were significantly lower than HCs, but were counter intuitively high (relative to HCs) during False-Moving and False-Still. LX showed a similarly low sense of ownership for the left hand, with responses below the HC CIs in all conditions, and never more than -1.0. Responses to control statement 1 were generally low (participants did not feel like they were looking directly at their hand), with LX falling inside the HC range. By contrast, LX's responses to control statement 2 were high (indicating a lack of position sense); she fell outside the HC range in all except the Veridical condition, in which HCs reported increased difficulty locating their hand.

\section{Discussion}

This paper provides the first direct examination of agency in parietal-type 
AHS. We predicted that damage to the posterior parietal lobe would impair veridical motor awareness and result in reduced self-reports of agency during active (volitional), goal-directed movements of the affected limb. In line with our prediction, LX reported abnormally low agency during the Veridical movement condition, for which HCs felt a strong sense of agency. However, her agency scores were higher than HCs in the False-Still and False-Moving conditions, for which HCs gave very low agency ratings. This result seems to partly contradict our initial prediction; however, it is important to note that in both instances LX did not report a strong sense of agency, with her responses being close to zero, and only slightly above those of HCs in the False-Moving condition. Movement without motor signals (i.e. Passive movement) elicited low ratings of agency that were no different from HCs (in line with our second prediction).

Our prediction that agency ratings would deviate from controls in all conditions except passive movement might, on first inspection, appear to contradict the idea that motor awareness arises from the detection of sensorimotor discrepancies or incongruence. Since such incongruence occurs only during certain (i.e. falsemoving and false-still) conditions in our experiment, one might expect that a deficit in motor awareness and abnormal agency ratings would occur only in these conditions. However, this prediction fails to take into account the fact that the posterior parietal lobe is involved in monitoring one's own movements, while parallel, low-level comparison of actual and predicted sensory information depends on premotor areas (Desmurget \& Sirigu, 2009; Sirigu et al., 2004). Thus, damage to the inferior parietal lobe might result in poor motor awareness and abnormal agency ratings even when low-level signals of sensorimotor incongruence are present, because such signals are not able to engage parietal areas responsible for conscious (veridical) motor 
awareness. Support for this explanation can be found in recent theoretical models of agency (Synofzik et al., 2008), which propose that agency involves a low-level feeling and a higher-level judgment of agency. What is more, this idea is supported by recent neuropsychological (e.g. Preston et al., 2010), neuroimaging (e.g. Kühn, Brass \& Haggard, 2013), and brain stimulation studies (e.g. Preston \& Newport, 2008; Weiss, Tsakiris, Haggard \& Schütz-Bosbach, 2014), which highlight the existence of explicit and implicit motor awareness processes. However, further research is needed to identify whether both low- and high-level motor awareness is impaired in patients with (parietal-type) AHS.

Overall, our results suggest that LX's parietal damage impaired veridical motor awareness and the sense of agency (Desmurget \& Sirigu, 2009). This conclusion is consistent with previous research connecting the parietal lobes to agency in both healthy (e.g. Farrer et al., 2003; Preston \& Newport, 2008) and braininjured individuals (e.g. Preston et al., 2010). Additionally, LX demonstrates that AHS is not always purely a deficit of motor control (Frith et al., 2000). Although AHS often involves a failure to inhibit actions elicited by external cues (Frith et al., 2000; Kritikos et al., 2005; Riddoch et al., 2001), in at least some instances (e.g. the parietal-type described here) AHS can involve abnormal agency (see Synofzik et al., 2008). In fact, damage to the SMA, which is a frequent cause of AHS, has been found to underlie an implicit sense of agency (see Kühn et al., 2013; Moore, Ruge, Wenke, Rothwell \& Haggard, 2010). However, we acknowledge that the number of questionnaire items and trials used in our study limit our conclusions. Also, it is possible that participants interpreted the questionnaire items in subtly different ways (e.g. questions regarding being in control of one limbs can be interpreted in a general sense [i.e. “was I capable of controlling the hand I was looking at?”] or specific sense 
[“was I actually controlling the hand I was looking at?”]), thereby increasing variability in the data. Further research is therefore needed to corroborate these findings in parietal and other subtypes of AHS, using various measures of agency (e.g. implicit and explicit measures; see Kühn et al., 2013; Preston et al., 2010; Weiss et al., 2014).

An interesting, additional finding or our study was that LX expressed consistently low ownership for her anarchic hand. Previous research identifies the inferior parietal lobe as part of an attentional network involved in stimulus-driven detection of behaviourally relevant stimuli (Corbetta \& Shulman, 2002), damage to which has been found in disorders of body ownership (Jenkinson, Haggard, Ferreira \& Fotopoulou, 2013). In healthy controls, evidence for a dissociation between agency and body ownership has been somewhat contentious, with some studies supporting the independence of these components (Kalckert \& Ehrsson, 2012; Tsakiris et al., 2010), while others suggest some degree of shared relationship (Tsakiris, Prabhu \& Haggard, 2006; Balslev, Nielsen, Lund, Law \& Paulson, 2006). Accounts of anarchic hand are equally controversial, as some researchers regard personification of the limb to indicate a deficit in ownership (Doody \& Jankovic, 1992), while others propose that personification reflects a selective deficit in agency and not ownership (Synofzik et al., 2008). Our results support the suggestion that agency and ownership are distinct processes, since changes in agency were observed independent of (stable albeit poor) ownership of the anarchic hand. Of further interest is the finding that, despite her low ratings of ownership for the affected hand, LX never explicitly denied ownership of the hand during clinical interviews (see 'case report' section, above). This finding might be explained in terms of the improved sensitivity of experimental measures in comparison to clinical interviews, particularly at discriminating between subtle 
dissociations. This suggestion is also consistent with the idea that (like agency) ownership comprises different low- and high-level processes: i.e., a non-conceptual feeling of ownership and a conceptual judgment of ownership (Synofzik et al., 2008). Unfortunately, we are unable to draw further conclusions regarding the role of ownership in AHS, given LX's atypical neuropathology and the limited assessment performed. Therefore, future research into AHS should also seek to experimentally examine different levels of body ownership in detail.

Possible criticisms of our study are that LX's ratings reflect a negative response bias, failure to give extreme ratings, or inability to make decisions. However, LX responded within the HC range for control statement 1, disagreeing with the assertion that she was looking directly at her hand rather than a video image. Her strong disagreement with this statement demonstrates her ability to make decisions and give extreme ratings, thereby negating these as explanations for her ambivalent agency ratings. Likewise, LX's agreement with control statement 2 ("it felt like I could not really tell where my hand was") shows willingness to provide extreme positive scores, thus negating a negative-response bias. These responses are also consistent with her poor position sense.

Finally, in addition to its role in motor awareness, the parietal cortex is known to play a key role in the integration of multisensory information relating to actions, via numerous connections with cortical and subcortical regions related to sensory and motor signals (Daprati, Sirigu \& Nico, 2010). Thus, concurrent sensory impairments arising from parietal damage may also contribute to LX's abnormal sense of agency and AHS. The importance of proprioceptive information in action recognition is highlighted by Farrer, Franck, Paillard and Jeannerod (2003), who found that neurologically healthy individuals were able to recognise movements as their own 
using only proprioceptive information (during passive movement), while, conversely, a patient with complete haptic deafferentation including loss of proprioception was unable to recognise her own movements. Interestingly, the deafferented patient showed a tendency to misidentify actions as being her own rather than deny that the movements were hers, suggesting that proprioceptive loss alone may increase the sense of agency. Further research is needed to disentangle the contribution of comorbid sensory complications in abnormal agency and AHS following parietal damage.

In conclusion, our study highlights the role of veridical motor awareness in parietal AHS, demonstrating, for the first time, impaired agency for volitional movements of the anarchic limb. Further experimental studies are needed to examine the phenomenology of parietal-type AHS in detail. Results from the type of experimental task described here should be compared across the three major varieties of AHS (i.e. parietal, frontal and callosal), and differences in performance between the anarchic and unaffected hands examined. Such studies will help develop a clear nosology of AHS and reveal the functional neuroanatomy of different subtypes. 


\section{References}

Assal, F., Schwartz, S., \& Vuilleumier, P. (2007). Moving with or without will: Functional neural correlates of alien hand syndrome. Annals of Neurology, 62, 301-306.

Balslev, D., Nielsen, F. A., Lund, T. E., Law, I., \& Paulson, O. B. (2006). Similar brain networks for detecting visuo-motor and visuo-proprioceptive synchrony. NeuroImage, 31(1), 308-312.

Berti, A., Bottini, G., Gandola, M., Pia, L., Smania, N., Stracciari, A., ... Paulesu, E. (2005). Shared cortical anatomy for motor awareness and motor control. Science, 309, 488-491.

Corbetta, M., \& Shulman, G. L. (2002). Control of goal-directed and stimulus-driven attention in the brain. Nature Reviews Neuroscience, 3, 201-215.

Daprati, E., Sirigu, A., \& Nico, D. (2010). Body and movement: Consciousness in the parietal lobes. Neuropsychologia, 48, 756-762.

Desmurget, M., \& Sirigu, A. (2009). A parietal-premotor network for movement intention and motor awareness. Trends in Cognitive Sciences, 13(10), 411-419.

DiCiccio, T. J., \& Efron, B. (1996). Bootstrap confidence intervals. Statistical Science, 11(3), 189-228.

Doody, R. S. \& Jankovic, J. (1992). The alien hand and related signs. Journal of Neurology, Neurosurgery, \& Psychiatry, 55, 806-810.

Farrer, C., Frank, N., Georgieff, N., Frith, C. D., Decety, J., \& Jeannerod, M. (2003). Modulating the experience of agency: A positron emission tomography study. NeuroImage, 18, 324-333.

Farrer, C., Franck, N., Paillard, J., \& Jeannerod, M. (2003). The role of proprioception in action recognition. Consciousness and Cognition, 12, 609-619. 
Farrer, C., \& Frith, C. D. (2002). Experiencing oneself vs another person as being the cause of an action: The neural correlates of the experience of agency. Neuroimage, 15(3), 596-603.

Frith, C. D., Blakemore, S-J., \& Wolpert, D. M. (2000). Abnormalities in the awareness and control of action. Philosophical Transactions of the Royal Society of London: Biological Sciences, 355, 1771-1788.

Graff-Radford, J., Rubin, M. N., Jones, D. T., Aksamit, A. J., Ahlskog, J. E., Knopman, D. S., ... Josephs, K. A. (2013). The alien limb phenomenon. Journal of neurology, 1-9.

Halligan, P. W., Marshall, J. C., \& Wade, D. T. (1989). Visuospatial neglect: Underlying factors and test sensitivity. The Lancet, 14, 908-911.

Jenkinson, P. M., Haggard, P., Ferreira, N. C., \& Fotopoulou, A. (2013). Body ownership and attention in the mirror: Insights from somatoparaphrenia and the rubber hand illusion. Neuropsychologia, 51(8), 1453-1462.

Kalckert, A. \& Ehrsson, H. H. (2012). Moving a rubber hand that feels like your own: a dissociation of ownership and agency. Frontier in Human Neuroscience. 6, 40.

Kritikos, A., Breen, N., \& Mattingley, J. B. (2005). Anarchic hand syndrome: Bimanual coordination and sensitivity to irrelevant information in unimanual reaches. Cognitive Brain Research, 24, 634-647.

Kühn, S., Brass, M., \& Haggard, P. (2013). Feeling in control: Neural correlates of experience of agency. Cortex, 49, 1935-1942.

Leube, D. T., Knoblich, G., Erb, M., \& Kircher, T. T. J. (2003). Observing one’s hand become anarchic: An fMRI study of action identification. Consciousness and Cognition, 12, 597-608. 
Marchetti, C., \& Della Sala, S. (1998). Disentangling the alien and anarchic hand. Cognitive Neuropsychiatry, 3(3), 191-207.

McIntosh, R. D., Brodie, E. E., Beschin, N., \& Robertson, I. H. (2000). Improving the clinical diagnosis of personal neglect: A reformulated comb and razor test. Cortex, 36, 289- 292.

Moore. J. W., Ruge, D., Wenke, D., Rothwell, J., \& Haggard, P. (2010). Disrupting the experience of control in the human brain: Pre-supplementary motor area contributes to the sense of agency. Proceedings of Biological Science, 277(1693), 2503-2509.

Nelson, H. E. \& Willison, J. (1991). National Adult Reading Test (NART): Test Manual. (Second ed.) Windsor, UK: NFER Nelson.

Preston, C., Jenkinson, P. M., \& Newport, R. (2010). Anosognosia for hemiplegia as a global deficit in motor awareness: Evidence from the non-paralysed limb. Neuropsychologia, 48(12): 3443-3450.

Preston, C., \& Newport, R. (2008). Misattribution of movement agency following right parietal TMS. Social Cognitive and Affective Neuroscience, 2(1): 26-32.

Riddoch, M. J., Humphreys, G. W., \& Edwards, M. G. (2001). An experimental analysis of unintentional lower limb action. Neuropsychologia, 39, 574 -579.

Scepkowski, L. A., \& Cronin-Golomb, A. (2003). The alien hand: Cases, categories, and anatomical correlates. Behavioral and Cognitive Neuroscience Reviews, 2(4), 261-277.

Sirigu, A., Daprati, E., Ciancia, S., Giraux, P., Nighoghossian, N., Posada, A., \& Haggard, P. (2004). Altered awareness of voluntary action after damage to the parietal cortex. Nature Neuroscience, 7(1), 80-84.

Synofzik, M., Vosgerau, G., \& Newen, A. (2008). I move, therefore I am: A new 
theoretical framework to investigate agency and ownership. Consciousness and Cognition, 17, 411-424.

Tsakiris, M., Prabhu, G., \& Haggard, P. (2006). Having a body versus moving your body: how agency structures body-ownership. Consciousness and Cognition, 15(2), 423-432.

Tsakiris, M., Longo, M. R., \& Haggard, P. (2010). Having a body versus moving your body: Neural signatures of agency and body-ownership. Neuropsychologia, 48(9), 2740-2749.

Wechsler, D. (1999). WASI: Wechsler Abbreviated Scale of Intelligence. San Antonio, TX: Harcourt Assessment.

Weiss, C., Tsakiris, M., Haggard, P., \& Schütz-Bosbach. (2014). Agency in the sensorimotor system and its relation to explicit action awareness.

Neuropsychologia, 52, 82-92. 


\section{Figure Captions}

Figure 1. a) An MRI showing the larger part of LX's lesion located in the right inferior parietal lobule; at the anterior margin the lesion encroaches into the superior temporal gyrus and angular gyrus, and at the posterior boundary there is some damage also traversing the occipito-parietal junction. A follow-up MRI (b) performed upon completion of the study shows LX's brain to be free from sclerotic lesions. All images are radiological convention (right shown on left side).

Figure 2. a) A schematic representation of the experimental set-up. The video camera was suspended from the centre of the tripod and connected to an LED display, located to the left of the real hand in an standard vertical orientation, to produce a personcentred view of the unseen hand as seen from above. Four experimental conditions (b) were created using this set-up via the manipulation of intention and visual feedback.

Figure 3. Questionnaire statements and responses for control statement 1 (panel a), control statement 2 (panel b), agency scores (panel c), and ownership scores (panel d). LX (crosses) fell within HCs (open circles) 95\% confidence intervals (error bars) for control statement 1 (a), while for control statement 2 (b) LX scored higher than HC 95\% confidence intervals for all conditions except Veridical movement. For the agency score (c) LX fell outside HC 95\% confidence intervals for all conditions except Passive. For the ownership score (d) LX scored lower than HCs 95\% confidence intervals in all conditions. 